\title{
Das türkische Welfare-Regime unter der AKP: vom konservativen südeuropäischen zum muslimischen Typus?
}

\author{
Noemi TRUCCO ${ }^{1}$ \\ Universität Fribourg
}

\begin{abstract}
Einleitung
\end{abstract}
Dieser Beitrag möchte das türkische Welfare-Regime unter der Partei für Gerechtigkeit und Fortschritt (Adalet ve Kalkınma Partisi, AKP) genauer betrachten. Spricht man von WelfareRegime, so ist damit meist eine spezifische Aufteilung der Produktion und Allokation von Wohlfahrt zwischen Staat, Markt und Familie gemeint (Esping-Andersen 1998:35-47). In muslimischen Gesellschaften weisen Welfare-Regimes normalerweise eine charakteristische Ähnlichkeit auf: Die Wohltätigkeit, die unter anderem auf der religiösen Pflicht der Almosensteuer (zakat) jedes Einzelnen beruht (Ali 2014:17-18) und dadurch unabhängig von Markt, Staat und Familie gedacht werden muss, spielt dort in der sozialen Sicherung eine grosse Rolle. Seekings zieht sogar einen spezifischen muslimischen Regime-Typus in Betracht (Seekings 2005:17). Obwohl die Türkei grösstenteils muslimisch ist, kann sie aufgrund von zwei historischen Besonderheiten nicht diesem Regime-Typus zugerechnet werden: zum einen ist sie laizistisch, zum anderen verfügt sie über sehr starke staatliche Strukturen (Dinç 2009:14). Diese beiden Besonderheiten haben das türkische Welfare-Regime geprägt.

Ein starker Staat bedeutet in diesem Kontext, dass der Staat als Akteur agiert, der die Gesellschaft von oben umformt und modernisiert (Keyman und Içduygu 2005:5). Dieses autoritäre Staatsverständnis, in dessen Rahmen die Interessen der Gesellschaft nicht durch einzelne Teile der Gesellschaft selbst definiert werden, sondern durch den Staatsapparat, setzte sich vom Osmanischen Reich in die 1923 gegründete Republik Türkei fort. Im Osmanischen Reich hatte keine Interessensvertretung der Bevölkerung in Form von Ständen oder ähnlichem bestanden. Zünfte existierten, aber diese standen unter vollständiger staatlicher Kontrolle. Dementsprechend wurde die politische, wirtschaftliche und gesellschaftliche Modernisierung im 19. Jahrhundert nicht von einem Bürgertum oder einer anderen nicht-staatlichen Gruppe initiiert, sondern von Eliten der Staatsadministration (Dinç 2009:17). Diese staatszentrierte Modernisierung wurde in der Republik Türkei bis zur Einführung des Mehrparteiensystems 1950 weitergeführt.

Ähnlich wie die starken staatlichen Strukturen hat auch der Laizismus seinen Ursprung im Osmanischen Reich: Da es im Islam keinen Klerus und keine Form der Institutionalisierung wie zum Beispiel im Falle der katholischen Kirche gibt, kam es zu einer Einbindung der sun-

\footnotetext{
${ }^{1}$ Die Autorin studiert Soziologie an der Universität Fribourg und Middle Eastern Studies an der Universität Bern und befindet sich derzeit im Masterstudiengang.
} 
nitischen Rechtsgelehrten (Ulema) in den Staat. Im Gegenzug setzte der Staat aufgrund seines Machtmonopols die orthodoxe sunnitische Auslegung des Islam durch und verfolgte heterodoxe Bewegungen (Dinç 2009:18, 46). Bei der Gründung der Republik Türkei konkurrierte die Ideologie der Staatselite mit dem Islam, weil beide eine Führung des Menschen nach bestimmten Regeln anstrebten. Es war für die Staatselite somit essentiell, die Macht des Islam zu beschränken. Der Islam wurde weiterhin in die staatlichen Strukturen inkorporiert, aber auch inhaltlich kontrolliert, unter anderem durch die Errichtung des Präsidiums für Religionsangelegenheiten (Diyanet İşleri Başkanlı̆̆ı) (Dinç 2009:49-50). Anders als die französische laïcité stellt der türkische Laizismus also keine vollständige Trennung von Kirche und Staat sowie eine konfessionelle Neutralität des Staates dar, sondern de facto einen sunnitischen Staatsislam (Dinç 2009:45).

Diese beiden historischen Besonderheiten wirkten sich auch auf die Sozialstaatsbildung im Osmanischen Reich und in der Republik Türkei aus. Dabei muss ein Aspekt erwähnt werden, der die starken staatlichen Strukturen mit der Wohlfahrt verbindet: Im Osmanischen Reich wurden Wohlfahrtsinstitutionen, seien es Spitäler, Suppenküchen oder wohltätige Fonds, durch religiöse Stiftungen (waqf) finanziert (Peri 1992:167). Stiftungen galten als freiwillige Form der Spenden (sadaqa), um Verdienste vor Gott zu erwerben. Die Gründung von Stiftungen stand ursprünglich nur dem Sultan und somit dem Staat zu (Quataert 2005:35). Die waqf dienten damit auch als Mittel zur Etablierung von Legitimität, zur Sicherung des Rückhalts in der Bevölkerung und zur Ausweitung von Einfluss (Singer 2009:135-137). Bis ins 18. Jahrhundert nahm der staatliche Einfluss soweit ab, dass es neben dem Sultan auch den Eliten wie z.B. dem Haushalt des Pascha oder des Wesirs möglich war, Stiftungen zu gründen (Quataert 2005:35). Weiter ist das Erbringen guter Taten im Islam Sache des Einzelnen und wird nicht von einer religiösen Instanz kontrolliert, sondern unterliegt der Verantwortung des Individuums. Dadurch institutionalisierte sich im Osmanischen Reich keine kollektive Idee von Wohltätigkeit. Es existierten keine religiösen Eliten, die sich mit sozialen Problemen und deren Lösung auseinandergesetzt hätten, und auch keine religiösen Wohlfahrtsverbände. Anders als in europäischen Gesellschaften flossen daher keine sozialpolitischen Vorstellungen religiöser Akteure in die Sozialstaatsbildung ein, denn deren Aktivität war auf den Bildungsbereich beschränkt (Dinç 2009:53, 90). Somit formulierte in der Republik Türkei der Staat im Alleingang die notwendigen Gesetze, „ohne vorher die betroffenen Adressaten (Unternehmer, Arbeiter) zu konsultieren“ (Dinç 2009:88).

Der vorliegende Beitrag möchte Reformen des türkischen Sozialstaats unter der AKPRegierung, die seit 2002 an der Macht ist und sich als islamisch-konservativ versteht, in den Vordergrund rücken. Dabei sollen insbesondere die Ausrichtung und die Werte der AKP näher betrachtet werden, um daraus Rückschlüsse auf Reformen in der Sozialpolitik zu ziehen. Werden in den Reformen unter der AKP Elemente sichtbar, die auf Verschiebungen hin zu einem muslimischen Welfare-Regime verweisen? Im ersten Teil des Beitrags soll der türkische Sozialstaat bis in die 1990er Jahre skizziert werden. Der zweite Teil fokussiert auf die AKP und der dritte Teil thematisiert die Reformen unter der AKP. 


\section{Die Entwicklung des türkischen Sozialstaats bis Ende der 1990er Jahre}

In der Analyse von Welfare-Regimes wird die Türkei oft dem südeuropäischen Typus zugeteilt (Gough 1996, Saraceno 2002:23), der ein stark fragmentiertes, hierarchisches und korporatistisches System aufweist, das für formell Angestellte - meist den männlichen Haupternährer - aufgrund des Erwerbsstatus Zuschüsse an die Gesundheitsversorgung und Renten vorsieht. Gleichzeitig existiert eine Arbeitsmarktstruktur, in welcher unbezahlte Care-Arbeit, informelle Erwerbstätigkeit - vor allem von jungen Erwachsenen, ehemaligen Langzeitarbeitslosen oder Marginalisierten - und Selbständigkeit sehr wichtig sind (Buğra und Keyder 2006:212; Yakut-Cakar 2007:103-104). Seekings nennt diesen Regime-Typus in seiner Untersuchung von südlichen Welfare-Regimes inegalitarian corporatist regime und definiert ihn durch „achieving income security through forms of risk-pooling and/or saving that are dependent on employment" (Seekings 2005:16). Das korporatistische Element ist sehr wichtig, das heisst, Ansprüche können aufgrund von Zugehörigkeit zu einer Berufsgruppe gestellt werden. Gleichzeitig ist dieses Regime fundamental inegalitär, weil gewisse Gruppen von formeller Beschäftigung praktisch ausgeschlossen sind (Seekings 2005:16-17). Die formelle Erwerbstätigkeit steht also im Zentrum und das System dient vor allem dem Statuserhalt der versicherten Erwerbstätigen und nicht primär dem universellen sozialen Ausgleich (Dinç 2009:85).

Ein Sozialstaat existierte in der Türkei von der Republiksgründung 1923 bis zum Ende des Zweiten Weltkriegs kaum (Dinç 2009:61), nur die Bildung war unentgeltlich (Eder 2010:157). Mit dem Ende des Einparteienregimes im Jahre 1946 und dem Übergang zu einem Mehrparteiensystem wurde ein begrenztes System der sozialen Sicherung entsprechend dem türkischen Modernisierungsverständnis eingeführt, d.h. die notwendigen Gesetze wurden von Beamten ohne innergesellschaftliche Debatten vom Ausland übernommen und implementiert (Dinç 2009:41). In der Literatur werden Macht- bzw. Systemwechsel als politische Gelegenheitsfenster zur Einführung sozialpolitischer Reformen begriffen², was in der Türkei auf die Einführung der ersten sozialstaatlichen Massnahmen sicherlich zutrifft. Das neue System der sozialen Sicherung umfasste die Rentenkasse der Türkischen Republik für Beamte (Emekli Sand $\imath \breve{g} \imath$ ) und die Sozialversicherungsanstalt für Arbeitnehmer (Iş̧̧i Sigortaları Kurumu). Beide richteten Renten- und Gesundheitsleistungen aus, deckten zusammen jedoch nur einen geringen Teil der türkischen Bevölkerung ab (Buğra und Candas 2011:518). Hier werden die bereits erwähnten korporatistischen und inegalitären Elemente offenkundig.

Nach dem Militärputsch 1960 wurde eine neue Verfassung ausgearbeitet, in welcher durch die Betonung des Rechts aller Bürger auf Bildung, Gesundheit und Arbeit zum ersten Mal eine sozialpolitische Verpflichtung des Staates eingeräumt wurde (Dinç 2009:62; Yakut-Cakar 2007:105). Gleichzeitig führte die Türkei mit US-Unterstützung importsubstituierte Industrialisierung (ISI) als Entwicklungsstrategie ein (Maxfield und Nolt 1990:72). Die Periode des ISIModells in der Türkei von den 1960er bis in die 1980er Jahre zeitigte hohe Profite, aber auch

\footnotetext{
${ }^{2}$ Vgl. dazu Sidney G. Tarrows Begriff political opportunity in Bezug auf soziale Bewegungen, der auf einem Konzept von Tilly aufbaut (Tarrow 2011), sowie die Definition von window of opportunity von Buse, Booth, Murindwa, Mwisongo und Harmer (2008:4): „The right moment, from the decision-makers' point of view, may present itself when they are needed to be seen to be taking action, and the political costs of doing so are likely to be low and the benefits potentially high".
} 
hohe Löhne zur Stimulation der Nachfrage und hatte eine Expansion des nationalen Marktes zur Folge (Bozkurt 2013:378; Yakut-Cakar 2007:105). Dabei weitete sich auch die Sozialversicherung auf grössere Teile der Bevölkerung aus, deckte jedoch noch längst nicht die gesamte Bevölkerung ab (Boratav und Özuğurlu 2006:175-176). So wurde 1971 eine Sozialversicherungsanstalt für Selbständige (Bağımsız Çalışanlar Kurumu, BAĞ KUR) eingeführt. Laut Buğra und Candas war es jedoch einigen der anvisierten Gruppen (z.B. Kleinbauern) nicht möglich, der Beitragszahlung nachzukommen, was deren Zugang zu Sozialleistungen beschränkte (Buğra und Candas 2011:518). Eine weitere Absicherung, die 1973 eingeführte beitragsfreie und bedürfnisgeprüfte Altersrente für Menschen über 65 Jahren, konnte nur von Menschen in Anspruch genommen werden, die nicht bereits eine andere Rente bezogen und deren Familienmitglieder keine Unterstützung leisten konnten (Dinç 2009:62-63).

Hier wird eine Besonderheit sichtbar, die dem südeuropäischen Welfare-Regime eigen ist: die wichtige Rolle der Familie in der Wohlfahrt. Die Familie übernimmt ein Teil der sozialstaatlichen Aufgaben, wobei der Staat ein traditionelles Haupternährer-Familienmodell und eine spezifische Arbeitsteilung im Haushalt voraussetzt (Dinç 2009:164). Frauen können dabei Leistungen (z.B. im Gesundheitsbereich) anhand des Status ihres Vaters oder Ehemannes beziehen (Kılıç 2008:492). In der Türkei wird die Familie als Stütze der sozialen Fürsorge und als Pflegedienstleister jedoch anders als in den meisten europäischen Ländern nicht durch familienspezifische Transferleistungen entlastet (Dinç 2009:88).

In den 1980er Jahren gab die Türkei aufgrund zunehmender Krisen das ISI-Modell auf und wandte sich einer exportorientierten Entwicklungsstrategie zu. Das neue Modell führte zwar zu einem gewaltigen Wachstum der Exporte (Bozkurt 2013:378), aber auch zu einer stärkeren Segmentierung des Arbeitsmarktes in Bezug auf formell und informell Beschäftigte und $\mathrm{zu}$ ansteigender Arbeitslosigkeit, da eine gleichzeitige Deregulierung und intensivierte Privatisierung eine geringere Anzahl an formell Beschäftigten und eine vermehrte Auslagerung von Teilen der Produktion an Subunternehmen zur Folge hatte (Buğra und Keyder 2006:220; Yakut-Cakar 2007:105). Der Militärputsch 1980 führte zur Unterdrückung der Gewerkschaften und linken Bewegungen, wodurch die Hauptlast der neuen Entwicklungsstrategie von den Arbeitern getragen werden musste (Bozkurt 2013:378).

Aufgrund unzureichender staatlicher Abdeckung hatten sich in der Türkei schon früh informelle Formen der sozialen Sicherung etabliert. Einerseits hielten Menschen, die vom Land in die Stadt migrierten, Verbindungen zum agrarisch geprägten Heimatort aufrecht, über welche sie Zuschüsse erhalten konnten. Netzwerke von Familien und Dorfbekannten in der Stadt dienten ausserdem der Suche nach Arbeit oder Unterkunft (Buğra und Keyder 2006:220). Eine weitere Form fand sich im irregulären Immobiliensektor, den sogenannten gecekond $u^{3}$ (Buğra 1998:306). Diese illegal angeeigneten Grundstücke wurden regelmässig staatlich legalisiert, was als Transferleistung an die ärmeren Bevölkerungsteile und als Ausgleich von Defiziten der formellen Umverteilungsmechanismen verstanden werden kann, wobei die Formalisierung oftmals auch im Austausch für Wählerstimmen stattfand (Bozkurt 2013:377; Buğra

\footnotetext{
${ }^{3}$ Gecekondu bedeutet wörtlich über Nacht gelandet und bezeichnet irreguläre Wohnformen auf besetztem, öffentlichem Land. Der Staat baute kaum billige Wohnungen, besass aber verhältnismässig viel ungenutzte Grundstücke, die illegal angeeignet und so für Wohnraum genutzt wurden (Buğra 1998:307-309; Buğra 2007:44). Gemäss Buğra (1998:309) existierten in Istanbul 1980 etwa 100’000 nicht autorisierte Gebäude.
} 
1998:307-308; Buğra 2007:44). Als eine dritte informelle Form kann die Solidarität innerhalb der Familie betrachtet werden (Buğra und Adar 2008:99). Alle drei Formen kamen Anfang der 1980er Jahre unter Druck, als die Türkei die starken Anreize für ISI beseitigte, ihr Handelsregime liberalisierte und die Wirtschaft damit stärker international ausrichtete (Krueger und Aktan 1992:173). Es kam zu einer neuen Kommodifizierung von Land und zur Stärkung von Eigentumsrechten, was die Legalisierung der gecekondu erschwerte oder verunmöglichte (Buğra und Keyder 2006:221-222; Buğra 2007:44). Weiter wurden staatliche Landwirtschaftssubventionen verringert und es wurde zunehmend schwierig, kleine Landwirtschaftsbetriebe aufrechtzuerhalten (Buğra und Candas 2011:519; Yakut-Cakar 2007:109). Ausserdem führte die Intensivierung des Konflikts zwischen dem kurdischen Bevölkerungsteil und dem türkischen Staat in den 1990er Jahren zu erzwungener Migration und zu einem Abbruch der (ökonomischen) Beziehung mit der kurdischen Heimatregion (Buğra und Keyder 2006:220). Zusätzlich wurde der Familienverband im urbanen Kontext mehrheitlich nuklearisiert, durch die Kleinfamilie ersetzt und die Rolle von Verwandtschaftsbeziehungen als Sicherheitsnetz erodierte (Buğra und Adar 2008:99).

Aufgrund des verstärkten Drucks auf die informellen Formen der sozialen Sicherung durch die Integration in die globale Marktwirtschaft wurde 1986 der Fonds zur Förderung der sozialen Hilfe und Solidarität (Sosyal Yardımlaşma ve Dayanışmayı Teşvik Fonu) ins Leben gerufen, der sich aus Lotterieeinnahmen, Autobahn- und Brückengebühren finanzierte und monetäre Beihilfen für Menschen in absoluter Armut bieten sollte (Buğra und Candas 2011:519; Dinç 2009:78). Dabei mussten Antragsteller bestimmte Bedingungen erfüllen, die sich jedoch jährlich änderten. Es bestand zudem kein Rechtsanspruch auf Leistungen (Dinç 2009:77). Buğra und Keyder verstehen die Etablierung dieses Fonds als Eingeständnis, dass es für Familien zunehmend schwieriger wurde, die ihnen zugesprochenen Aufgaben innerhalb des Welfare-Regimes leisten zu können. Ausserdem erwähnt wird, dass mit dem Fonds die Hoffnung verbunden war, unter der Leitung und Initiative des Staates private Spenden zu erhalten, um das Budget zu entlasten, wobei die Vermischung von privaten und öffentlichen Geldern innerhalb eines Fonds eine typische Eigenschaft wohltätiger osmanischer Stiftungen (waqf) gewesen war, auf die nun Bezug genommen werden konnte. Dementsprechend wurde der Fonds als eine Dachorganisation von über 900 lokalen Stiftungen konzipiert (Buğra und Keyder 2006:222). Die AKP erkannte später als erste das Potential dieser Art von Wohlfahrtseinrichtung und machte Gebrauch davon (Buğra und Candas 2011:520). Darauf soll im dritten Teil dieses Beitrags vertieft eingegangen werden.

1990 wurde die nach Bedürftigkeit vergebene Grüne Karte im Gesundheitswesen eingeführt, die Zugang zu kostenloser medizinischer Betreuung gewähren sollte, wobei die Inanspruchnahme aufgrund des komplizierten Verfahrens zur Beantragung relativ niedrig blieb. Dennoch stellt die Grüne Karte einen Schritt hin zur Anerkennung des Rechts auf Gesundheitsversorgung dar (Buğra und Candas 2011:520).

Der türkische Sozialstaat, wie er bis Ende der 1990er Jahre existierte, wies somit mehrere Probleme auf. Das grösste war die Finanzierung des Sozialversicherungssystems: das Haushaltsdefizit der drei Sozialversicherungskassen machte ca. $4.5 \%$ des BIP aus ${ }^{4}$. Der türkische

\footnotetext{
${ }^{4}$ Der Durchschnitt von ca. 4.5\% des BIP über die 1990er Jahre berechnet Dinç (2009:91) aus den Angaben zu den einzelnen Jahren im Bericht der Fachkommission für Sozialschutz (T.C. Devlet Planlama Teşkilatı 2001:72-74).
} 
Staatshaushalt wurde dadurch stark belastet, weil das System durch Transfers aus dem Staatshaushalt vor dem Zusammenbruch bewahrt werden musste (Dinç 2009:91). Ein Teil der informell Erwerbstätigen hatte Zugang zum Gesundheitswesen als Mitversicherte eines formell erwerbstätigen Familienmitglieds, was das System stark beanspruchte (Buğra und Keyder 2006:217). Darüber hinaus sanken die Einnahmen, weil es Probleme gab, die Beiträge einzutreiben, betrogen wurde (z.B. durch die Angabe falscher Einkommen) und kein Mindestalter für die Pensionierung existierte, was zu vielen Frührentnern führte ${ }^{5}$. Der Anteil von Beitragszahlenden im Verhältnis zu Bezügern nahm kontinuierlich ab, obwohl die Türkei über eine relativ junge Bevölkerung verfügt. 2005 finanzierten noch 1,7 Erwerbstätige einen nicht (mehr) Erwerbstätigen. Ausserdem bedeuteten drei verschiedene Sozialversicherungskassen für drei Berufsklassen (Beamte, Arbeitnehmer, Selbständige) unterschiedliche Beiträge und Leistungen für verschiedene Berufsgruppen und damit viel Verwaltungsaufwand und höhere Kosten (Dinç 2009:92-94).

Auch die Veränderung der türkischen Arbeitsmarktstruktur durch die verstärkte Einbindung in den internationalen Handel in den 1980er Jahren spielte eine grosse Rolle. Sie führte, wie bereits angemerkt, zu abnehmenden Erwerbsmöglichkeiten im formellen Sektor und dadurch zu stark ansteigender Arbeitslosigkeit (Yakut-Cakar 2007:105, 125) sowie zu einem informellen Sektor mit einem Anteil von über 50\% der gesamten Erwerbstätigkeit (Buğra und Keyder 2006:217). Ein System der sozialen Sicherung, das so stark auf die formelle Anstellung setzte wie das türkische, konnte dadurch kaum alle Bürger ausreichend absichern (YakutCakar 2007:105). Insbesondere Frauen waren entweder nicht erwerbstätig - wozu auch die Mitarbeit als unbezahlte Familienangehörige gerechnet wird - oder sie arbeiteten häufig unter irregulären Arbeitsbedingungen in kleinen Werkstätten oder im Akkord zu Hause zu sehr tiefen Löhnen (Buğra und Keyder 2005:27; Kılıç 2008:494). Die weibliche Arbeitsmarktpartizipation war in der Türkei vergleichsweise niedrig und lag 2003 bei 28.1\% (Buğra und Keyder 2006:217-218). Dies entspricht dem vom Staat propagierten Familienbild: einem männlichen Haupternährer und einer geschlechtsspezifischen Arbeitsteilung im Haushalt (Dinç 2009:164).

Dies alles führte zu neuen Formen von vor allem urbaner Armut, insbesondere working poor (Buğra 2007:45). Der Anteil extremer Armut in der Türkei war gemäss Buğra und Keyder (2005:20) vernachlässigbar. Dafür lag die Arbeits-Armutsgefährdungsquote ${ }^{6}$ laut Eurostat in der Türkei 2007 bei 17.9\% (Eurostat 2016:o. S.). Dabei waren die öffentlichen Ausgaben des türkischen Staates für Arbeitslose, sozialen Wohnungsbau, Familienzulagen, Witwenund Waisenrenten u.ä. im Vergleich mit den OECD-Ländern sehr tief: sie lagen im Jahre 2008 bei 1,3\% des BIP, während der OECD-Durchschnitt 7,9\% ausmachte. Das deutet darauf hin, dass formell Beschäftigte zwar gut abgesichert wurden - denn dort lagen die prozentualen BIP-Anteile der Türkei im OECD-Durchschnitt -, die vom formellen Arbeitsmarkt Ausgeschlossenen jedoch vernachlässigt wurden (Buğra und Adar 2008:95-97).

Es ist nicht erstaunlich, dass es aufgrund dieser Probleme zu mehreren Reformen des Sozialstaats kam. Das weitaus wichtigste Reformpaket wurde unter der AKP initiiert und 2008

\footnotetext{
${ }^{5}$ Es wird geschätzt, dass 62\% der Rentner im Jahr 2009 Frührentner waren (Dinç 2009:92).

${ }^{6}$ „Anteil von Personen mit einem verfügbaren Äquivalenzeinkommen unter der Armutsgefährdungsschwelle, die auf 60\% des nationalen verfügbaren Median-Äquivalenzeinkommens (nach Sozialleistungen) festgelegt ist“ (Eurostat 2016:o.S.).
} 
implementiert. Im nächsten Teil soll erst die AKP, ihre Werte und Ausrichtung genauer betrachtet werden, bevor dann in einem dritten Teil die Reformen näher untersucht werden.

\section{Die AKP}

Die nationalen Wahlen vom November 2002 gewann die AKP, die zuvor noch nie an Wahlen teilgenommen hatte, überraschend mit 34.6\% der Stimmen und erhielt eine Mehrheit der Parlamentssitze (Gumuscu und Sert 2009:959). Viele ihrer Vorsitzenden wie Recep Tayyip Erdoğan oder Abdullah Gül waren bereits bekannt, entstammt die Partei doch einer Tradition islamisch-konservativer Parteien (Mecham 2004:339). In der postkemalistischen Türkei der 1950er Jahre wurden religiöse Interessen vor allem von konservativen Parteien bedient, eine islamisch-konservative Partei entwickelte sich erst relativ spät (Dinç 2009:52). Die erste politische Partei mit islamischer Orientierung war die Nationale Ordnungspartei (Millî Nizam Partisi) unter Necmettin Erbakan, die jedoch 1971, ein Jahr nach ihrer Gründung, bereits wieder verboten wurde. Aus dieser Partei gingen mehrere Folgeparteien hervor, die alle denselben ideologischen Ansatz teilten: die von Erbakan initiierte Nationale Sicht (Millî Görüş) (Gumuscu und Sert 2009:953) ${ }^{7}$. Die Millî Görüş-Bewegung vertritt islamische bzw. islamistische und türkisch-nationalistische Werte, hat eine anti-westliche Haltung (Hale und Özbudun 2010:5-7) ${ }^{8}$ und den Standpunkt, dass sich das Individuum der Gemeinschaft unterordnen muss (Gumuscu und Sert 2009:955). Als laizistischer Staat, dessen Verfassung religiöse Parteien explizit untersagt, verbot die Türkei die meisten der Folgeparteien (Gumuscu und Sert 2009:954; Hale und Özbudun 2010:5). Wichtig für die Entwicklung hin zur AKP war vor allem die Wohlfahrtspartei (Refah Partisi), die 1995 erneut unter Erbakan die Wahlen mit 21\% der Stimmen gewann und 1996 mit der Partei des Rechten Weges (Doğru Yol Partisi) eine Koalitionsregierung formte. Im Februar 1997 kam es jedoch zu einem sogenannten postmodernen Coup ${ }^{9}$, in dessen Folge Premierminister Erbakan im Juni 1997 zurücktreten musste (Gumuscu und Sert 2009:953). Kurz darauf wurde eine Spaltung innerhalb der Millî GörüşBewegung sichtbar, als sich eine stärker auf Demokratie, Menschenrechte und Pluralismus ausgerichtete Gruppe gegen die alte Führung der Bewegung aussprach und daraufhin eine eigene Partei gründete: die AKP, die Partei für Gerechtigkeit und Fortschritt (Adalet ve Kalkınma Partisi) (Gumuscu und Sert 2009:954).

Grundsätzlich findet sich in der Literatur zur AKP seit 2002 entweder ein verhalten positiver Unterton oder ein Misstrauen, was die Haltung der Partei zu Demokratie, Menschenrechten, Rechtsgrundlagen, Pluralismus, Säkularismus usw. betrifft (vgl. z.B. Aydın-Düzgit und

\footnotetext{
${ }^{7}$ Dabei weisen Gumuscu und Sert daraufhin, dass in millî, das eigentlich national bedeutet, sowohl die Bedeutung national als auch religiös bzw. muslimisch/islamisch mitschwingt (Gumuscu und Sert 2009:966, Fussnote 1). Diese Wortwahl erklärt sich aus dem legalen und konstitutionellen Verbot jeglicher anti-säkularer Aktivität politischer Parteien (Hale und Özbudun 2010:5).

${ }^{8}$ Die Millî Görüş-Bewegung existiert auch in Deutschland, siehe Schiffauer 2010.

${ }^{9}$ Dabei unterbreitete der politisch-militärisch zusammengesetzte Nationale Sicherheitsrat Erbakan am 28. Februar 1997 ein Dokument mit Massnahmen gegen die zunehmenden Aktivitäten politisch-islamistischer Gruppierungen, das dieser zu unterzeichnen hatte. Das hatte ein hartes Vorgehen gegen den politischen Islam und Erbakans Rücktritt zur Folge. Nach dem Rücktritt verbot das Verfassungsgericht die Wohlfahrtspartei und untersagte Erbakan, weiter politisch tätig zu sein (Gumuscu und Sert 2009:953).
} 
Keyman 2012, Gumuscu und Sert 2009, Hale und Özbudun 2010, Seckinelgin 2016). Nach dem Wiederaufflammen des Konflikts mit der kurdischen Bevölkerung Mitte 2015, mit den repressiven Massnahmen der Regierung gegenüber Journalisten, Opposition und Justiz (Amnesty International 2016) und dem Referendum im April 2017, das Erdoğan unbegrenzte Machtbefugnisse ermöglicht, scheint sich nun eher zweitere Perspektive zu bestätigen. Aspekte, die in der vor 2015 erschienenen Literatur zur AKP noch als positiv hervorgehoben werden, sich nun aber verändert haben, wie zum Beispiel, dass die AKP Wert auf freie Meinungsäusserung lege (Gumuscu und Sert 2009:20), werden in diesem Beitrag somit nicht einbezogen, denn eine Analyse und Diskussion der Richtungsänderung der Politik der Partei oder ihres zunehmend autoritären Regierungsstils würde den Rahmen bei weitem sprengen. Es sollen somit vor allem die grundsätzliche Ausrichtung und Werte der AKP diskutiert werden, die seit 2002 mehr oder weniger konstant blieben und die vermutlich auch in die Ausgestaltung sozialpolitischer Massnahmen und Reformen eingeflossen sind.

Das ökonomische Parteiprogramm der AKP ist auf eine freie Marktwirtschaft ausgerichtet und weist dem Staat - entgegen der türkischen Tradition eines eingreifenden, starken Staates - im wirtschaftlichen Bereich nur eine regulatorische und überwachende Funktion zu (Hale und Özbudun 2010:23). Die Türkei war nach der Wirtschaftskrise 2001 einige haushaltspolitische Verpflichtungen mit dem IWF und der Weltbank eingegangen, um Kredite zu erhalten. Die AKP bekannte sich nach Amtsantritt zum Programm des IWF zur makroökonomischen Stabilisierung der Türkei (Bozkurt 2013:373; Dinç 2009:92). Insbesondere diese liberale Ausrichtung hat der AKP die Unterstützung einer gläubigen, islamisch-konservativen Mittelschicht - so zum Beispiel des islamisch-konservativen Unternehmerverbandes MÜSIAD (Müstakil Sanayici ve İş Adamları Derneği) - und damit eine starke politische Basis gesichert. Diese Mittelschicht profitierte ursprünglich von der Öffnung der türkischen Wirtschaft in den 1980er Jahren (Gumuscu und Sert 2009:966), wurde danach aber durch die galoppierende Inflation und die Rezession im Jahre 2001 zunehmend unzufrieden (Mecham 2004:340). Interessanter Weise konnte die AKP ihre Wählerschaft nach der globalen Krise des Jahres 2008, die in der Türkei vor allem kleine und mittlere Unternehmen und damit vor allem die nicht und wenig gebildete Arbeiterschaft traf, sogar noch erweitern und holte 2011 in den nationalen Wahlen über 50\% der Stimmen. Umfragen deuteten darauf hin, dass es der AKP dabei gelang, sich die Unterstützung eines grossen Teils der Arbeiterschaft zu sichern, insbesondere derjenigen, die vom formellen System der sozialen Sicherung ausgeschlossen waren (Bozkurt 2013:373-374). Dass der Wahlerfolg der AKP auch mit sozialpolitischen Massnahmen zusammenhängen könnte, soll im nächsten Teil näher betrachtet werden.

Die AKP distanziert sich selbst von der Millî Görüş-Bewegung und beschreibt sich als konservative Partei (Hale und Özbudun 2010:20). Konservatismus betont grundsätzlich den Schutz von Institutionen wie der Familie und die Wichtigkeit von Traditionen zur Aufrechterhaltung der sozialen Ordnung. Der symbolische Gehalt des Konservatismus wird durch Religion bestimmt, in der Türkei also durch den sunnitischen Islam (Bozkurt 2013:382). Aus der Perspektive der AKP kreiert Religion eine natürliche moralische und soziale Ordnung (Mecham 2004:351). Diese Einstellung ist gut ersichtlich, bezieht sich die AKP doch in ihrem Diskurs stetig auf den Islam, sobald es um den Erhalt von Institutionen und Werten geht. Ausserdem evoziert sie das Bild des Staates als Grossfamilie mit gemeinsamen geteilten Wer- 
ten (Bozkurt 2013:382). Die Partei selbst definiert Konservatismus als eine evolutionäre im Gegensatz zu einer revolutionären Auffassung von Wandel und als Top-down-Projekt der gesellschaftlichen Umformung (Social Engineering) ${ }^{10}$, wobei Traditionen, Normen und Werte der Gesellschaft gewürdigt und geschützt und nur graduell Veränderungen im bestehenden System vorgenommen werden sollen. Dabei versäumte es die AKP allerdings, eine Grenze zwischen Islamismus und dem von ihr betonten Konservatismus zu ziehen, was Kritikern den Anlass gegeben hat, der Partei eine versteckt islamistische Agenda zu unterstellen. Ihre konservative Identität scheint sie somit weit weniger erfolgreich konstruieren zu können als ihre ökonomische (Gumuscu und Sert 2009:965-966).

Eine weitere wichtige Komponente in der Ausrichtung der AKP ist ihr nationalistisches Element. Dabei wird eine türkisch-kulturelle Einheit imaginiert ${ }^{11}$, die über ethnische Grenzen hinausgeht und über religiöse (sunnitisch-islamische) Werte und Traditionen zusammengehalten wird. Insbesondere dem osmanischen Erbe wird dabei identitätsstiftende Wirkung zugeschrieben (Bozkurt 2013:383) - was die Partei mit der Millî Görüş-Bewegung teilt und im Gegensatz zur kemalistischen Anschauung steht (Hale und Özbudun 2010:6-7). Ausserdem verfügt die AKP über eine starke Grassroots-Organisation mit Fokus auf Gemeinden und Nachbarschaften, welche sie von der Wohlfahrtspartei übernommen hat (Mecham 2004:343, 351). Das ist eine relativ typische Strategie islamischer, aber auch islamistischer Organisationen wie z.B. der Muslimbrüder in Ägypten, um sich die politische Unterstützung der von sozialer Exklusion betroffenen Bevölkerung über sozialpolitische Massnahmen zu sichern (Manea 2016:14).

Die AKP kann zusammengefasst als islamisch-konservative Partei mit stark nationalistischen Elementen und ökonomisch liberaler Ausrichtung beschrieben werden. Im nächsten Teil werden die sozialpolitischen Massnahmen und Reformen unter der AKP näher betrachtet.

\section{Sozialstaatliche Reformen unter der AKP}

Unter der AKP-Regierung wurden umfangreiche Reformen des Sozialstaats eingeleitet, die sich sowohl auf die beitragsfinanzierten Sozialversicherungen als auch auf die steuerfinanzierte soziale Fürsorge erstreckten (Dinç 2009:96; Kılıç 2008:487). Laut Buğra und Keyder betrafen die Reformpläne im Kern zwei Punkte: die Finanzierung des Sozialstaates und die Herausforderungen neuer Armut (Buğra und Keyder 2006:213).

Im Gesundheitswesen umfasste die Reform nebst anderem die Einführung einer beitragsfinanzierten allgemeinen Krankenversicherung (Genel Sağlık Sigortası) und die schrittweise Abschaffung der Grünen Karte bis 2010, wobei die Kassenbeiträge für Menschen, deren Ein-

\footnotetext{
${ }^{10}$ Dabei muss erwähnt werden, dass die Perspektive von gesellschaftlicher Veränderung Top-down, einhergehend mit einer starken Betonung der Rolle der Elite, und die Idee von Social Engineering seit Beginn des 20. Jahrhunderts im Osmanischen Reich und in der darauffolgenden Republik Türkei unter den politischen Eliten weit verbreitet waren und sind. So vertrat u.a. der einflussreiche türkische Soziologe Ziya Gökalp diese Position (Parla 1985).

${ }^{11}$ Seit dem Niedergang des multiethnischen und multireligiösen Osmanischen Reichs ab Mitte des 19. Jahrhunderts ist die Herstellung und Konstruktion einer (wie auch immer gearteten) Einheit ein kontinuierlich wiederkehrendes Thema. Dies zu vertiefen, würde den Rahmen dieses Beitrags aber sprengen.
} 
kommen unter einer bestimmten Einkommensgrenze liegt, vom Staat bezahlt werden sollen (Buğra und Keyder 2006:216; Dinç 2009:101-102). Der angestrebte allgemeine Zugang zum Gesundheitssystem wird als wesentlicher Punkt der Reformen angesehen, aber die Finanzierung über Beiträge und nicht über Steuern war umstritten. Aufgrund des hohen Anteils an informell Beschäftigten wurde angenommen, dass ein Grossteil derjenigen, die nach einer Bedürftigkeitsprüfung Beiträge zahlen müssten, keine Möglichkeit hat, dieser Pflicht nachzukommen. Nebst einem verwehrten Zugang zum Gesundheitssystem wären diese Menschen aufgrund nicht bezahlter Beiträge dann auch gesetzlichen Sanktionen unterworfen. Die Beitragsfinanzierung wurde vor allem von der Regierung, Wirtschaftskreisen und insbesondere auch der Weltbank befürwortet, nicht jedoch vom IWF, da der Staat die Beiträge für die Armen übernehmen muss und damit die Kosten für den Staat eher zu- denn abnehmen (Adar 2007:168; Bozkurt 2013:386-387; Kılıç 2008:491-492). Interessanter Weise stammen die meisten Beiträge zur Gesundheitsreform aus der Zeit kurz vor oder nach der Implementierung, so dass innerhalb der Literatur noch kaum Hinweise zu finden sind, ob die kontrovers diskutierten Punkte auch tatsächlich eingetroffen sind oder nicht.

Ein anderes, sehr wichtiges Element der Reformen war die Vereinigung der verschiedenen Sozialversicherungen unter der Behörde für soziale Sicherheit (Sosyal Güvenlik Kurulu, SGK) im Ministerium für Arbeit und soziale Sicherheit (Buğra und Adar 2008:101; Buğra und Keyder 2006:215). Dabei wurde eine Gleichbehandlung aller Berufsgruppen angestrebt ${ }^{12}$, das Rentenalter für Frauen und Männer auf 65 Jahre angehoben und die Beitragsdauer für die Alters- und Invalidenrente verlängert. Obwohl die Regierung vor allem die Aufhebung der Ungleichbehandlung der Berufsgruppen hervorhob, diente diese Massnahme hauptsächlich der Kostensenkung, weil der Staat damit nicht mehr für die medizinische Behandlung und die Lohnfortzahlung der Beamten bei Krankheit, Arbeitsunfall oder Schwangerschaft aufkommen muss. Beamte zahlen durch die Reform nun gleiche Beiträge wie alle anderen Berufsgruppen (Dinç 2009:105). Man kann diesen Teil der Reformen als Versuch werten, das stark korporatistische Element des türkischen Sozialstaats aufzuheben oder zumindest abzuschwächen.

Ein weiterer Teil der Reformen umfasste die Vereinigung der bisherigen steuerfinanzierten Sozialhilfeprogramme unter dem Direktorat für Sozialhilfe (Sosyal Yardım Müdürlüğ̈̈), weil die Verwaltungsstrukturen so umgestaltet werden sollten, dass beitragsfinanzierte und steuerfinanzierte Leistungen behördlich getrennt werden, um mehr Effizienz zu gewährleisten. Andere Sozialhilfeprogramme wie monetäre Beihilfen für Familien mit behinderten Kindern blieben jedoch weiterhin eigenständig bestehen. Die vor den Reformen bestehende Pluralität der sozialen Fürsorge, so urteilt Dinç, wurde somit nicht aufgehoben. Ausserdem wurden die steuerfinanzierten Leistungen der sozialen Fürsorge nicht weiter ausgebaut, sondern blieben bestehen wie zuvor (Dinç 2009:102-105).

\section{Die Rolle der Familie}

Betrachtet man die Reformen genauer, so fällt vor allem auf, dass die AKP die relativ starke Rolle der Familie im türkischen Welfare-Regime nicht aufgehoben oder durch zusätzliche Unterstützungen entlastet hat, weil sich die Reformen hauptsächlich auf die Finanzierungs-

\footnotetext{
${ }^{12}$ Traditionell wurden in der Türkei Beamte bevorzugt behandelt (Dinç 2009:89).
} 
probleme der Sozialversicherungen konzentrierten, aber z.B. die öffentliche Pflege nicht ausgebaut wurde (Dinç 2009:106). Im offiziellen sozialpolitischen Diskurs wird die Familie noch immer als zentrale Institution innerhalb des Welfare-Regimes betrachtet (Buğra und Keyder 2006:222). Dem Staat wird dabei eine die Familie unterstützende Rolle zugewiesen, während jene den sozialen Schutz des Einzelnen sicherstellen soll (Buğra und Keyder 2006:212). Die Pflege und Betreuung von Kindern, Behinderten und Senioren wird der Familie überlassen und nicht durch Transferleistungen ausgeglichen; mit der Ausnahme von Witwen- und Waisenrenten (Dinç 2009:155-156). Der türkische Sozialstaat ermöglicht es jungen Erwachsenen (z.B. Studierenden) aufgrund unzureichender Beihilfen auch nicht, sich ökonomisch unabhängig zu machen. Er bringt nicht nur junge Erwachsene dazu, länger im Haushalt ihrer Eltern zu leben, sondern forciert Haushalte auch, ältere und pflegebedürftige Familienmitglieder aufzunehmen (Dinç 2009:164). Wie bereits erwähnt, ist die Kleinfamilie und nicht mehr der Familienverband die Norm, die durch diese sozialpolitische Arbeitsteilung zwischen Staat und Haushalt immer stärker unter Druck gerät, wobei die Care-Arbeit hauptsächlich zu Lasten der Frauen geht (Buğra und Keyder 2006:221). Daneben federn Haushalte auch die Folgen der Arbeitsmarktstruktur mit einem hohen Anteil an informeller Erwerbstätigkeit ab. Dadurch, dass Familiensolidarität als gegeben angesehen wird - denn eine Vernachlässigung der familiären Unterstützung wird gesellschaftlich abgelehnt -, ist es bestimmten Bevölkerungsgruppen möglich, eine informelle und/oder prekäre Beschäftigung aufzunehmen, da der Familienhaushalt die unzureichende Absicherung bei Arbeitslosigkeit auffängt und unzureichende Einkommen ergänzt. Damit stehen dem informellen Sektor gleichzeitig stets genügend Arbeitskräfte zur Verfügung (Dinç 2009:151-155, 169).

Es handelt sich bei der Familiensolidarität um einen tief verankerten Wert, für den sich die AKP einsetzt, wenn sie familienorientierte Politik priorisiert und die Bedeutung familiärer Netzwerke betont (Buğra und Candas 2011:521). Dabei stehen die Erhaltung der traditionellen, sozialen Ordnung und der Schutz der Familie im Mittelpunkt (Bozkurt 2013:382). In ihrem Diskurs zur Familie und der Rolle der Frau wird die konservativ-paternalistische, aber auch islamisch-religiöse Ausrichtung der AKP augenscheinlich. So hat Premierminister Erdoğan z.B. Familien höchstpersönlich dazu aufgefordert, mindestens drei Kinder zu haben und greift damit sehr stark in das Privatleben seiner Bevölkerung ein (Seckinelgin 2016:271).

\section{Restrukturierung: Kostensenkung, Workfare und Flexibilisierung der Arbeitsbeziehungen}

Die Reformen unter der AKP konzentrieren sich vor allem auf das Finanzierungsproblem, indem im Sozialversicherungssystem Kosten gesenkt, Einnahmen erhöht und die zuvor bestehenden drei Kassen fusioniert wurden (Dinç 2009:105-106). Gleichzeitig wurde aber eine Krankenversicherung mit angestrebtem universellem Zugang eingeführt. Damit kam man dem Ziel der Kostensenkung eigentlich nicht nach, da der Staat die Kosten für die arme Bevölkerung übernehmen muss und deswegen die staatlichen Kosten eher zu- denn abnehmen. Allerdings können nicht bezahlte Beiträge gesetzlich sanktioniert werden (Bozkurt 2013:387; Kılıç 2008:492). Überdies wurden alle steuerfinanzierten Leistungen nicht weiter ausgebaut (Dinç 2009:106). In diesen Massnahmen wird die ökonomische Ausrichtung der AKP sichtbar: sie hatte sich sich bereits 2002 dazu bekannt, dass sie die Rolle des Staates vor allem darin sieht, den freien Wettbewerb auf dem Markt zu garantieren und Hindernisse für die Privat- 
wirtschaft zu beseitigen, und unterstützte von Anfang an die Privatisierung des noch stark staatlichen Industrie- und Dienstleistungssektors (Hale und Özbudun 2010:100).

Dabei fördert die AKP-Regierung auch Mikrokredit-Modelle. Die AKP ist der liberalen Auffassung, dass soziale Unterstützung unter der Bedingung produktiver Tätigkeit gewährt werden soll (Buğra und Keyder 2006:223). Sie sieht Mikrokredite als beste Möglichkeit der Armutsverminderung an und vertritt die Perspektive, dass öffentliche Transferleistungen die Armut nur erhöhen, da sie Arbeitsanreize reduzieren und Abhängigkeiten hervorbringen (Buğra und Candas 2011:522). Diese Verschiebung von welfare zu workfare entspricht voll und ganz der ökonomischen Ausrichtung der Partei. Es existieren sehr wohl Schattenseiten des Mikrokredit-Modells: Mikrokredite werden oft eingesetzt, um die weibliche Erwerbsquote zu erhöhen, was jedoch darin resultieren kann, dass Frauen zu Hause Güter in Akkordarbeit unter dem Marktpreis herstellen. Dazu kommt, dass in der Türkei ein Gesetz diese Frauen als Selbständige betrachtet, womit ihre Ansprüche eingeschränkt werden, obwohl sie eher als abhängige Subunternehmende angesehen werden müssten (Kılıç 2008:498-499).

Die angestrebte ökonomische Restrukturierung wird weiter deutlich sichtbar in der Flexibilisierung der Arbeitgeber-/Arbeitnehmerbeziehungen, da ab 2003 Akkordarbeit, Zeitarbeit und insbesondere das Subunternehmertum legalisiert wurden (Bozkurt 2013:391). Die Effekte dieser Flexibilisierung versucht die AKP durch Sozialhilfeprogramme auszugleichen, deren Anzahl stark zugenommen und die zunehmend als Ersatz für andere Funktionen des Sozialstaats eingesetzt werden, wobei die Regierung begann, gewisse dieser Programme in den privaten Sektor auszulagern (Bozkurt 2013:374).

\section{Private Wohltätigkeit unter der AKP}

Die AKP propagiert private Wohltätigkeit zur Armutsverminderung, wobei sie sich islamischer Elemente bedient, um zivilgesellschaftliche Initiativen $\mathrm{zu}$ diesem Zweck anzuregen (Buğra und Adar 2008:102; Buğra und Keyder 2006:224). Dem Staat wird die Rolle als Partner wohltätiger zivilgesellschaftlicher Initiativen zugewiesen, statt ihn als Akteur innerhalb einer Logik der Besteuerung und Umverteilung zu sehen (Buğra und Candas 2011:518). Dabei werden gewisse, ursprünglich staatliche Funktionen wohltätigen Gruppen und Vereinen übergeben oder gleich ganz in den privaten Sektor ausgelagert (Bozkurt 2013:374). Die Grenzen zwischen dem Staat, dem privaten Sektor und der Zivilgesellschaft werden dabei verwischt (Buğra und Candas 2011:517). Der Staat verzichtet zum Teil sogar auf Steuereinnahmen, um die privaten Anteile in der Wohlfahrt auszubauen, wie zum Beispiel im Jahre 2005 im Fall der Kampagne „100\% Unterstützung für Bildung“ (Eğitime \%100 Destek), in deren Rahmen privaten Spendern eine hunderprozentige Steuerermässigung auf ihre Spenden zugesagt wurde (Eder 2010:173). Laut Buğra und Candas hat die AKP den Diskurs islamischer Philanthropie erfolgreicher eingesetzt als alle politischen Akteure zuvor. Sie merken jedoch an, dass die Verschiebung hin zu privater Wohltätigkeit sowohl in Übereinstimmung mit islamischer Wohltätigkeit als auch mit den aktuellen Trends in der globalen Sozialpolitik steht (Buğra und Candas 2011:521-522).

Der bereits zur Sprache gebrachte Fonds zur Förderung der sozialen Hilfe und Solidarität (Sosyal Yardımlaşma ve Dayanışmayı Teşvik Fonu), eine Dachorganisation von über 900 Stiftungen, wurde unter der AKP im Jahre 2004 in ein Direktorat umgewandelt (Sosyal Yardım- 
laşma ve Dayanışma Genel Müdürlüğü, SYDGM) (Buğra und Candas 2011:521; Buğra und Keyder 2006:222). Bemerkenswert daran war laut Eder vor allem, dass das Direktorat einen viel grösseren Grad an Autonomie erhielt und einen grossen Anteil seiner Ressourcen nun aus Fonds ausserhalb des Budgets bezieht, welche keine parlamentarische Freigabe benötigen. Das Direktorat untersteht nicht mehr der öffentlichen Prüfung, sondern nur noch dem Premierminister. Die Mittel, die das SYDGM den ihm unterstellten Stiftungen zukommen liess, stiegen zwischen 2004 und 2008 von 400 Mio. Lira auf 1.25 Mia. Lira.

Eder (2010:174-175) weist dabei vor allem darauf hin, dass die ausserbudgetären Quellen sowie die fehlende öffentliche Überprüfung das Potential für Klientelismus und die Möglichkeit des Gebrauchs für politische Zwecke massiv erhöht hat (unabhängig von der Wirksamkeit der sozialpolitischen Massnahmen der unterstützten Stiftungen). So stiegen die Ausgaben vor den lokalen Wahlen im Jahre 2009 signifikant an und in Tunceli wurden durch eine vom SYDGM unterstützte Stiftung sogar Waschmaschinen und Geschirrspüler an potentielle Wähler verteilt. Die unabhängige Behörde, welche die Wahlen überprüfte, intervenierte, um diese Praxis zu stoppen. Den Provinzgouverneur interessierte das nicht, er erhielt direkte Unterstützung von Premierminister Erdoğan (Buğra und Candas 2011:523). Dieser Zusammenhang zwischen Wahlzyklen und höheren Sozialausgaben wird in der Literatur diskutiert (vergleiche zum Beispiel Block 2002 oder Niles 1999). Dabei ist es im Falle der türkischen Wahlen auf Gemeindeebene im Jahre 2009 sicherlich so, dass sozialpolitische Massnahmen bewusst eingesetzt wurden, um eine Wiederwahl zu erreichen, wie das Beispiel Tunceli zeigt. Eder (2010:182) interpretiert das Zugeständnis an das SYDGM, ausserbudgetäre Mittel verwenden zu können und das Direktorat der öffentlichen Prüfung zu entziehen, als Ausweitung der politischen Macht und Einflusssphäre der AKP.

Das SYDGM ist jedoch nicht der einzige Bereich, in dem sozialpolitische Massnahmen Klientelismus und politische Instrumentalisierung ermöglichen. Unter der AKP haben Gemeinderegierungen mehr Verantwortung in der Zuteilung von Sozialhilfe erhalten (Bozkurt 2013:389), was der Grassroots-Organisation der AKP entspricht. Viele der Mittel auf Gemeindeebene stammen nicht aus den Gemeindebudgets, sondern wiederum aus privaten Spenden. Dabei besteht einerseits das Problem, dass Sozialhilfe aus privaten Spenden nicht konstant und deswegen enorm unzuverlässig ist, und andererseits die Vergabe meist intransparent, willkürlich und subjektiv gehandhabt wird. Ausserdem können Spenden als Bestechung genutzt werden - so können sich Behörden gegen wohltätige Spenden dazu bereit erklären, nicht juristisch gegen ein illegal tätiges Unternehmen vorzugehen, oder sie können spezifische Privilegien vergeben (Buğra und Keyder 2006:224; Eder 2010:177-179). Überdies begann die AKP auch, NGOs Spendensammlungen ohne vorherige staatliche Bewilligung zu erlauben. Eder (2010:180) sieht dies als Ausdruck dafür, dass die AKP auch NGOs als neue Instrumente der staatlichen Politik betrachtet. Eine dieser NGOs namens Der Leuchtturm (Deniz Feneri) wurde in Deutschland aufgrund von Spendenbetrug verurteilt, weil Mittel irregulär für ökonomische und politische Interessen islamistischer Kreise eingesetzt wurden, wobei dazu auch Mitglieder der AKP gehören sollen (Buğra und Candas 2011:524).

Auch im Mikrokredit-Modell wird der Versuch sichtbar, die für soziale Sicherung zuständigen Akteure durch eine Verschiebung hin zu privater Wohltätigkeit neu zu definieren (Buğra und Adar 2008:93). Im Falle der Mikrokredit-Systeme in der Türkei werden diese 
nämlich sowohl durch den Staat, als auch durch wohltätige Organisationen oder durch eine Partnerschaft beider implementiert (Buğra und Candas 2011:523).

\section{Schlussfolgerungen}

Zusammengefasst können die sozialpolitischen Reformen unter der AKP folgendermassen interpretiert werden: Einerseits wird versucht, mit der Reform der Finanzierung und der Vereinheitlichung des Sozialversicherungssystems den Sozialschutz weiter zu gewährleisten (Dinç 2009:106). Dies entspricht der liberalen Wirtschaftspolitik der Partei und führt weg vom ursprünglichen, inegalitären Korporatismus des türkischen Sozialstaats (Buğra und Keyder 2006:227). Die starke Rolle der Familie innerhalb des Welfare-Regimes wurde jedoch weiterhin aufrechterhalten, ohne sie zu entlasten, was dem sehr traditionell-konservativen Familienbild der AKP entspricht (Dinç 2009:106). Ihre konservativ-paternalistische Ausrichtung hält die AKP aber nicht unter allen Umständen aufrecht: So war sie bereit, den von ihr hochgehaltenen Schutz der Frauen abzuschwächen, indem sie z.B. das Nachtarbeitsverbot für Frauen aufhob, weil es der Flexibilisierung des Marktes diente (Kılıç 2008:496-497). Die Reformen sind somit vor allem von ihrer wirtschaftlichen Ausrichtung geprägt.

Andererseits nahmen Sozialhilfeprogramme zur Abfederung der Folgen der Flexibilisierung der Arbeitgeber-Arbeitnehmerbeziehungen stark zu, allerdings hat die Partei es ausgezeichnet verstanden, private Wohltätigkeit anzuregen und viele dieser Programme in den privaten Sektor auszulagern (Bozkurt 2013:374). Dies kann als konservativ-liberale Tendenz begriffen werden, den Abbau von staatlichen Leistungen mit islamischen Auffassungen von Wohlfahrt zu ergänzen (Buğra und Keyder 2006:226). Dabei macht die AKP einen ideologischen Rückgriff auf ein osmanisches Erbe - die waqf - und betont dabei deren islamisches Element, was ihrer islamisch-religiösen Ausrichtung entspricht. So wie die Stiftungen im Osmanischen Reich werden die Privatisierungstendenzen unter der AKP von Eder (2010:156) als Versuch der Ausweitung des Einflusses interpretiert: nicht nur als Rückzug des Staates, sondern vielmehr auch als Machtausbau in weiteren Sphären des sozialen und ökonomischen Lebens, denn die Auslagerung und Privatisierung gewisser Angebote bietet zunehmend Möglichkeiten für Klientelismus und politische Instrumentalisierung der sozialpolitischen Massnahmen. Weil in diesen Sozialhilfeprogrammen zum Teil ausserbudgetäre Mittel verwendet werden, die nicht der öffentlichen Prüfung und parlamentarischen Freigabe unterstehen, wird die Macht der regierenden politischen Elite ausgebaut (Eder 2010:182).

Die Sozialpolitik unter der AKP ist somit eindeutig Ausdruck ihrer islamischkonservativen und wirtschaftsliberalen Ideologie. Vor allem aber begreift und nutzt die AKP Sozialpolitik als Instrument zum Machterhalt und -ausbau. Die Türkei nähert sich aufgrund der Förderung der privaten Wohltätigkeit unabhängig von Markt, Staat und Familie durch die AKP nun somit teilweise dem von Seekings beschriebenen muslimischen Welfare-Regime an. 


\section{Literaturverzeichnis}

Adar, Sinem (2007). Turkey: reform in social security. Journal of European Social Policy, 17(2), 167168.

Ali, Jan A. (2014). Zakat and Poverty in Islam. In: Clarke, Matthew und David Tittensor (Hg.). Islam and Development. Exploring the Invisible Aid Economy (15-32). Farnham: Ashgate.

Amnesty International (2016). Turkey. In: Amnesty International (Hg.). Amnesty International Report 2015/2016. The State of the World's Human Rights (369-372). London: Amnesty International.

Aydın-Düzgit, Senem and Keyman, Fuat (2012). EU-Turkey relations and the stagnation of Turkish democracy. Sabanc1 University ID:Working Paper 02.

Block, Steven A. (2002). Political business cycles, democratization, and economic reform: the case of Africa. Journal of Development Economics, 67, 205-228.

Boratav, Korkut und Metin Özuğurlu (2006). Social Policies and Distributional Dynamics in Turkey: 1923-2002. In: Karshenas, Massoud und Valentine M. Moghadam. Social Policy in the Middle East. Economic, Political, and Gender Dynamics (156-189). Basingstoke: Palgrave Macmillan.

Bozkurt, Umut (2013). Neoliberalism with a Human Face: Making Sense of the Justice and Development Party's Neoliberal Populism in Turkey. Science \& Society, 77(3), 372-396.

Buğra, Ayşe (1998). The Immoral Economy of Housing in Turkey. International Journal of Urban and Regional Research 22(2), 303-307.

Buğra, Ayşe (2007). Poverty and Citizenship: An Overview of the Social-Policy Environment in Republican Turkey. International Journal of Middle East Studies, 39(1), 33-52.

Buğra, Ayşe und Sinem Adar (2008). Social Policy Change in Countries without Mature Welfare States: The Case of Turkey. New Perspectives on Turkey, 38, 83-106.

Buğra, Ayşe und Aysen Candas (2011). Change and Continuity under an Eclectic Social Security Regime: The Case of Turkey. Middle Eastern Studies, 47(3), 515-528.

Buğra, Ayşe und Çaglar Keyder (2005). Poverty and Social Policy in Contemporary Turkey. Boğaziçi University Social Policy Forum, January 2005. Zugriff am 18.12.2016 auf http://www.spf.boun.edu.tr/docs/WP-Bugra-Keyder.pdf.

Buğra, Ayşe und Çaglar Keyder (2006). The Turkish welfare regime in transformation. Journal of European Social Policy, 16(3), 211-228.

Buse, Kent, David Booth, Grace Murindwa, Aziza Mwisongo und Andrew Harmer (2008). Donors and the Political Dimensions of Health Sector Reform: The Cases of Tanzania and Uganda. Working Paper 7. London: Overseas Development Institute.

Dinç, Cüneyd (2009). Sozialstaat als Produkt einer Staatselite. Die Türkei im südeuropäischen Vergleich. Wiesbaden: VS Verlag für Sozialwissenschaften. 
Eder, Mine (2010). Retreating State? Political Economy of Welfare Regime Change in Turkey. Middle East Law and Governance, 2, 152-184.

Esping-Andersen, Gøsta (1998). Die drei Welten des Wohlfahrtskapitalismus. Zur Politischen Ökonomie des Wohlfahrtsstaates. In: Lessenich, Stephan und Ilona Ostner (Hg.). Welten des Wohlfahrtskapitalismus. Der Sozialstaat in vergleichender Perspektive (19-56). Frankfurt: Campus Verlag.

Eurostat (2016). Arbeits-Armutsgefährdungsquote - EU-SILC Erhebung. Internet-Seite. Zugriff am 18.12.2016 auf http://ec.europa.eu/eurostat/tgm/table.do?tab=table\&init=1\&language=de\&pcode=tesov1 10\&plugi $\mathrm{n}=1$

Gough, Ian (1996). Social assistance in Southern Europe. South European Society and Politics, 1(1), 123.

Gumuscu, Sebnem und Deniz Sert (2009). The Power of the Devout Bourgeoisie: The Case of the Justice and Development Party in Turkey. Middle Eastern Studies, 45(6), 953-968.

Hale, William und Ergun Özbudun (2010). Islamism, Democracy and Liberalism in Turkey. The case of the AKP. Oxon: Routledge.

Keyman, E. Fuat und Ahmet Içduygu (2005). Introduction. Citizenship, identity and the question of democracy in Turkey. In: Keyman, E. Fuat und Ahmet Içduygu (Hg.). Citizenship in a Global World. European questions and Turkish experiences (1-27). Abingdon: Routledge.

Kilıç, Azer (2008). The Gender Dimension of Social Policy Reform in Turkey: Towards Equal Citizenship? Social Policy \& Administration, 42(5), 487-503.

Krueger, Anne O. und Okan H. Aktan (1992). Swimming against the Tide. Turkish Trade Reform in the 1980s. San Francisco: International Center for Economic Growth.

Manea, Elham (2016). Islamism in Ideology and Context. Unveröffentlichte Vorlesungsfolien 15.03.16. Institut für Islamwissenschaft und Neuere Orientalische Philologie, Universität Bern, Bern.

Maxfield, Sylvia und James H. Nolt (1990). Protectionism and the Internationalization of Capital: U.S. Sponsorship of Import Substitution Industrialization in the Philippines, Turkey and Argentina. International Studies Quarterly, 34(1), 49-81.

Mecham, Quinn R. (2004). From the ashes of virtue, a promise of light: the transformation of political Islam in Turkey. Third World Quarterly, 25(2), 339-358.

Niles, Kimberley J. (1999). Economic Adjustment and Targeted Social Spending: The Role of Political Institutions. Paper prepared for the World Development Report 2001 meetings, August 16-17, Castle Donington. Zugriff am 18.12.2016 auf http://siteresources.worldbank.org/INTPOVERTY/Resources/WDR/DfiDProject-Papers/niles.pdf.

Parla, Taha (1985). The social and political thought of Ziya Gökalp 1876 -1924. Leiden: E. J. Brill. 
Peri, Oded (1992). Waqf and Ottoman Welfare Policy. The Poor Kitchen of Hasseki Sultan in Eighteenth-Century Jerusalem. Journal of the Economic and Social History of the Orient, 35(2), 167-186.

Quataert, Donald (2005). The Ottoman Empire, 1700-1922 (2. Auflage). Cambridge: Cambridge University Press.

Saraceno, Chiara (2002). Introduction: exploring social assistance dynamics. In: Saraceno, Chiara (Hg.). Social Assistance Dynamics in Europe. National and local poverty regimes (1-34). Bristol: The Policy Press.

Schiffauer, Werner (2010). Nach dem Islamismus. Die Islamische Gemeinschaft Milli Görüş. Eine Ethnographie. Berlin: Suhrkamp Verlag.

Seckinelgin, Hakan (2016). Social policy and conflict: the Gezi Park-Taksim demonstrations and uses of social policy for reimagining Turkey. Third World Quarterly, 37(2), 264-280.

Seekings, Jeremy (2005). Prospects for Basic Income in Developing Countries: A Comparative Analysis of Welfare Regimes in the South. CSSR Working Paper No. 104. Kapstadt: Centre for Social Science Research.

Singer, Amy (2009). Charity. In: Ágoston, Gábor und Bruce Masters (Hg.). Encyclopedia of the Ottoman Empire (135-138). New York: Facts on File.

Tarrow, Sidney G. (2011). Power in Movement. Social Movements and Contentious Politics (dritte, überarbeitete Ausgabe). Cambridge: Cambridge University Press.

T.C. Devlet Planlama Teşkilatı (2001). Sosyal Güvenlik Özel Ihtisas Komisyonu Raporu. Ankara: T.C. Devlet Planlama Teşkilatı.

Yakut-Cakar, Burcu (2007). Turkey. In: Deacon, Bob und Paul Stubbs. Social Policy and International Interventions in South East Europe (103-129). Cheltenham: Edward Elgar. 